\title{
Serviços ecossistêmicos prestados pelos manguezais do Rio Grande do Norte (nordeste do Brasil)
}

\author{
Diógenes Félix da Silva Costa ${ }^{(a)}$ \\ ${ }^{(a)}$ Departamento de Geografia/Grupo de Pesquisa em Geoecologia e Biogeografia de Ambientes Tropicais, \\ Universidade Federal do Rio Grande do Norte, E-mail: diogenes.costa@ pq.cnpq.br
}

\section{EIXO: BIOGEOGRAFIA, MANEJO DE ÁREAS NATURAIS E PROTEGIDAS: CONSERVAÇÃO DA BIODIVERSIDADE}

\begin{abstract}
Resumo
Os manguezaissão ecossistemas que promovem uma variedade de bens e serviços tangíveis e intangíveis para o bem estar das comunidades humanas, onde a subestimação destes serviços contribue para expandir as perdas e a degradação destes ecossistemas. Esta pesquisa em questão teve por objetivo identificar e analisar os bens e serviços prestados pelos ecossistemas de manguezal encontrados ao longo dos principais sistemas estuarinos do Estado do Rio Grande do Norte (NordesteBrasil). O sistema de diagrama resultou em um modelo qualitativo com estruturas montadas em forma de ação e reação. A dinâmica agregada de uso humano e seus reflexos nos processos ecológicos do manguezal, a partir da recuperação dos manguezais, ampliando-se a área ocupada pela vegetação desse ecossistema, tem-se um melhor desempenho dos processos ecológicos, os quais propiciam a manutenção ou maior disponibilidade dos serviços prestados.
\end{abstract}

Palavras chave: Gestão costeira;Áreas úmidas; Biogeografia de manguezais; Etnoecologia.

\section{Introdução}

A exploração dos recursos do manguezal em economias tradicionais de subsistência é usualmente não intensiva. Todavia, o marcado aumento da interação e modernização dessas economias tem levado a uma exploração intensiva dos manguezais e às vezes a sua remoção (GRASSO, 1998). Esses ecossistemas promovem uma variedade de produtos e serviços. Segundo Grasso e Schaeffer-Novelli (1999), a subestimação dos seus valores totais e dos impactos das atividades humanas são os maiores fatores que contribuem para expandir as perdas e a degradação dos ecossistemas de manguezal.

Já para Motta (2006), outro problema chave quando se trata da valoração ambiental é o alto grau de interconectividade com e entre os ecossistemas, dificultando a predição de como os gestores deverão proceder. Os sistemas naturais desenvolvem uma série de funções, todavia existem muitas interconexões como, por exemplo, muitas das funções de produção são dependentes da "fixação de energia solar e produção de biomassa", "estocagem e reciclagem de matéria orgânica" e "estocagem e reciclagem de nutrientes". O conceito de "serviço ecossistêmico" vem evoluindo ao longo dos últimos anos, podendo ser entendido 
como os benefícios que os ecossistemas proporcionam direta e indiretamente (tangíveis e intangíveis) para o uso e bem estar humano (GROOT et al., 2002, 2010; FISHER; TURNER; MORLING, 2009; HAINES-YOUNG; POTSCHIN, 2013; COSTANZA et al., 2014).

Ao longo do globo, os ecossistemas de manguezal são altamente reconhecidos por atuarem como áreas de berçário para espécies importantes comercialmente, podendo contribuir também para a produtividade marinha em alto mar através do fluxo de detritos (PRIMAVERA, 2004). Esses ecossistemas também promovem uma série de serviços ecossistêmicos, como por exemplo a estabilização dos sedimentos suspensos na água, os quais são geralmente anóxicos. Para as espécies vegetais, esse problema do suprimento de ar para as raízes é resolvido graças a um sistema de raízes aéreas que, em parte, determina a estrutura física do ecossistema (LUGO; SNEDAKER, 1974). Para Kjerfve e Lacerda (1993), é justamente esse sistema de raízes que retarda o fluxo de água, onde essa resistência dissipa a energia durante as fortes marés de sizígia, serviço atualmente importante para os ocupantes das margens dos estuários (cidades, fazendas de camarão, salinas, comunidades de pescadores etc.).

Segundo Gilbert e Jansen (1998), esse serviço de controle de erosão também propicia a criação de um ambiente calmo, permitindo a estabilização dos sedimentos e inibindo sua (re)suspensão. A estabilização de sedimentos pelos manguezais é de fundamental importância para a proteção da linha de costa e, por conseqüência, para o desenvolvimento das atividades humanas aí realizadas.

Juntamente com os serviços comentados acima, os ecossistemas de manguezal também atuam como sumidouro de substâncias químicas dissolvidas e suspensas na água, removidas durante o fluxo das marés, assim como pelos organismos que vivem junto às raízes da vegetação. Este serviço de sumidouro abarca desde fertilizantes e pesticidas lançados às águas dos estuários, juntamente com os esgotos das empresas situadas nas suas margens, enquadrando esse serviço como de alta relevância. (LACERDA, 1993; RAMOS E SILVA et al., 2007).

Considerando-se a necessidade de preservação e a importância ecológica dos manguezais frente ao intenso processo de uso e ocupação da zona costeira da Região Nordeste do Brasil (e.g. expansão imobiliária, carcinicultura, salinicultura), a pesquisa em questão teve por objetivo identificar e analisar os bens e serviços prestados pelos ecossistemas de manguezal encontrados ao longo dos principais sistemas estuarinos do Estado do Rio Grande do Norte. Esta área está localizada entre as latitudes 440' e 6³0'S e longitudes $34^{\circ} 50^{\prime}$ e $38^{\circ} 30^{\prime} \mathrm{W}$ e com aproximadamente $380 \mathrm{~km}$ de extensão (Figura 1), apresentando um trecho oriental com clima tropical sub-úmido e intensamente ocupado pela carcinicultura, enquanto que o trecho setentrional é submetido a secas periódicas, onde a salinidade torna-se um fator de estresse, acarretando na formação de extensas planícies hipersalinas (apicuns), áreas cujo solo apresenta elevados 
teores de sais e que são inundadas apenas durante as preamares (SCHAEFFER-NOVELLI et al., 2000). Nos estuários em análise, estas planícies se tornaram zonas importantes do ponto de vista econômico, pois foram ocupadas por salinas solares para produção de sal marinho via evaporação solar (MAIA; LACERDA, 2005).

\section{Material e métodos}

Como estratégia metodológica, foi tomado como base o trabalho de Gilbert e Janssen (1998), que analisam os processos ecológicos dos manguezais e as interações humanas com estes ecossistemas através de um sistema de diagramas a fim de identificar e analisar as potenciais inter-relações, bem como integrar os processos ecológicos e o funcionamento dos ecossistemas à eficiência econômica dos sistemas estudados por economistas. Estes sistemas de diagramas foram adaptados para identificar e acessar os produtos e serviços ecossistêmicos gerados pelo sistema ambiental (ecossistema de manguezal) aos usuários das margens dos estuários do litoral do Rio Grande do Norte.

Em termos sequenciais, realizou-se a identificação dos bens e serviços ecossistêmicos através da seguinte sequência de atividades: 1) identificação dos bens e serviços in situ através da técnica check list, 2) classificação dos serviços ecossistêmicos com base nos estudos de Fisher, Turner e Morling (2009), De Groot et al. (2010), Costanza et al. (2014), utilizando-se o sistemaCommon International Classification of Ecosystem Services - CICES (HAINES-YOUNG; POTSCHIN, 2013; MAES; CROSSMAN; BURKHARD, 2016). Posteriormente, procedeu-se a descrição das retroalimentações e inter-relações, inferidos direta e indiretamente, utilizando sistemas de diagramas para identificar os produtos e serviços gerados pelos ecossistemas de manguezal, adaptando-se a proposta de Gilbert e Janssen (1998). Para esta última etapa, os diagramas foram elaborados com auxílio do software CorelDRAW Graphics Suite (32bit) X7 (@CorelDRAW). 


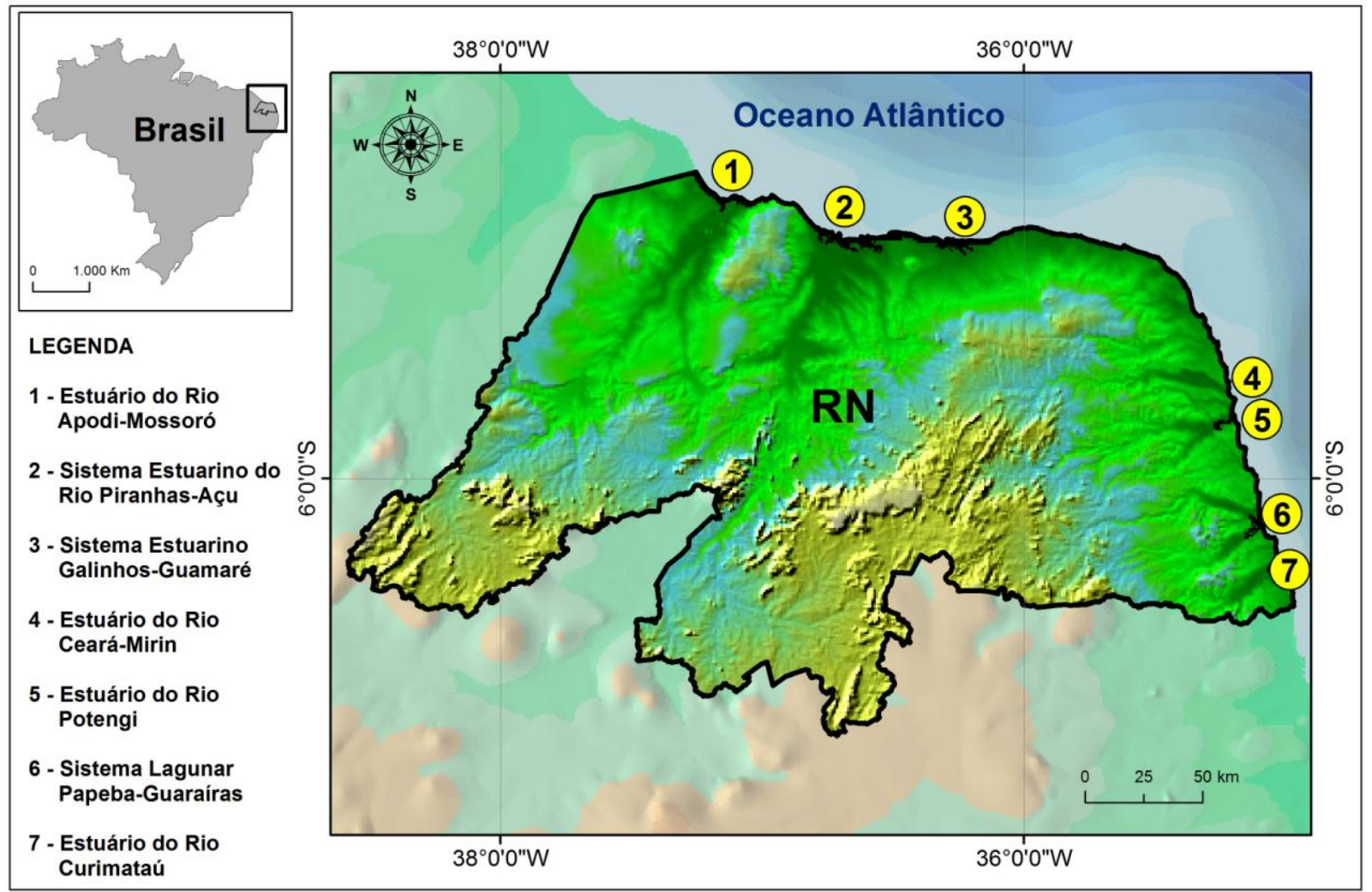

Figura 1 - Localização dos principais sistemas estuarinos do estado do Rio Grande do Norte.

O sistema de diagrama apresentado abaixo buscou exemplificar uma abordagem integrada das interações (direta e indiretas) entre a ação antrópica, os processos ecológicos e os serviços ecossistêmicos analisados, apresentando como resultado um modelo qualitativo com estruturas montadas em forma de ação e reação (Figura 2). Os diagramas usados representam formas básicas e são apresentados em sistemas de blocos para análise, ilustrando estoques, fluxos e outras variáveis. Juntamente com estes, são adicionados os produtos e serviços ecossistêmicos, assim como variáveis problema.

Em um primeiro momento, foram gerados sistemas de diagramas, os quais tiveram sua elaboração a partir das inter-relações entendidas entre o ecossistema de manguezal e as ações humanas (Figura 3). Nestes, a qualidade da cobertura do manguezal deriva dos processos ecológicos (Figuras 04), os quais controlam o desempenho dos serviços prestados pelo ecossistema. As figuras usam um formato simples de movimentação da esquerda para a direita: processos ecológicos, funções ambientais, produtos e serviços ecossistêmicos, e problemas ambientais. As funções ambientais, juntamente com os produtos e serviços promovidos são apresentados em combinações de estoque e fluxo. Nesse contexto, entende-se um "problema ambiental" no ecossistema como sendo um aumento da demanda por algum serviço, 
excedendo seu suprimento. Por conseguinte, esse problema pode ocasionar retroalimentação o com o ecossistema, ou gerar custos através das inter-relações entre os sistemas ecológicos e econômicos.

Para facilitar a compreensão dos blocos gerados seguir-se-á os símbolos utilizados porGilbert e Jansen (1998) (Figura 2).

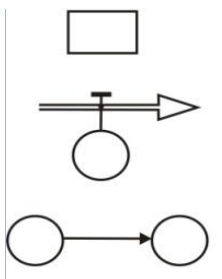

Estocado ou estado variável

Adicionamento ou subtração

do estoque

Variável b é influenciada

pela variável $a: \quad b=f(a)$

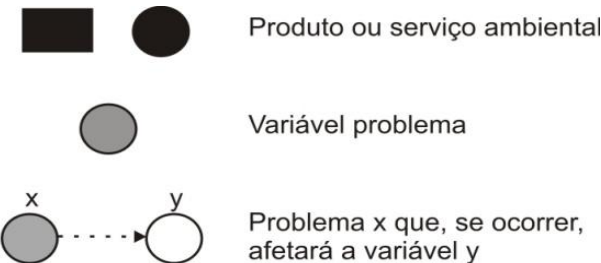

afetará a variável y

Figura 2 - Explanação dos símbolos utilizados nos diagramas.

Fonte: Traduzido de Gilbert e Jansen (1998).

\section{Resultados e Discussão}

Duas categorias de qualidades dos manguezais (saudável e degradado) são postas como indicadoras da resposta dos processos ecológicos do ecossistema frente as ações antrópicas negativas ou positivas, como a degradação e recuperação gerando dinâmicas distintas entre eles (Figura 4). Os processos comprometidos explicitam os danos em caso de retirada do manguezal gerados em virtude do desenvolvimento das atividades humanas (e.g. portos, fazendas de camarão, salinas, etc.). Historicamente, este fato tem ocasionado o surgimento de áreas degradadas, principalmente nos projetos de aquacultura e salinas abandonadas, onde a rentabilidade desses empreendimentos não justifica os custos ambientais incorridos com a remoção do manguezal.

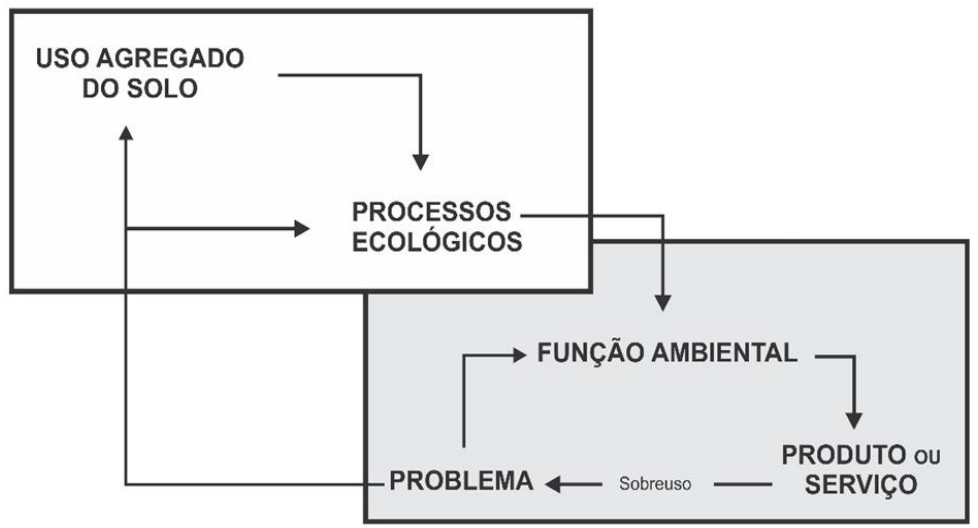

Figura 03 - Estrutura do sistema de diagramas.

Fonte: Adaptado a partir de Gilbert \& Jansen (1998). 
Analisando-se a dinâmica agregada de uso humano e seus reflexos nos processos ecológicos do manguezal (Figura 4), a partir da recuperação dos manguezais, ampliando-se a área ocupada pela vegetação desse ecossistema, tem-se um melhor desempenho dos processos ecológicos, os quais propiciam a manutenção ou maior disponibilidade dos serviços prestados. A saúde da comunidade vegetal tem uma influência direta em duas variáveis chaves (processos ecológicos) do ecossistema produtividade e estrutura física, juntamente com outros processos ecológicos. A variável "produtividade" é um agregado de estoques e fluxos associados com a fixação de carbono, juntamente com a estocagem e/ou reciclagem de nutrientes, fato este corroborado pela alta produtividade dos manguezais (RAMOS E SILVA et al., 2006; 2007; WALTERS et al., 2008).

A "produtividade" também está diretamente relacionada com o fato de o ecossistema de manguezal apresentar hábitats com abundante alimentação para espécies residentes temporárias, como espécies aquáticas juvenis ou adultas. Em outra perspectiva, também evidencia-se a disponibilização do serviço de Manutenção, no que diz respeito à reciclagem dos resíduos líquidos das águas que percorrem o ecossistema. As águas dos estuários do litoral Nordeste do Brasil são sobrecarregadas de esgotos domésticos e industriais, somados aos descartes das fazendas de camarão situadas nas zonas estuarinas. Este serviço de processamento biológico e reciclagem desses efluentes lançados na água do estuário já vem sendo amplamente ratificado em várias áreas do globo, representando um elemento chave para a gestão costeira, a ser altamente ponderado quando da formulação de projetos que impliquem diretamente da supressão de áreas de manguezal (RAMOS E SILVA et al.,2010; MARCHAND et al., 2012; ANDRADI-BROWN et al., 2013; MAITI; CHOWDHURY, 2013;LEE et al.,2014; MUKHERJEE et al., 2014). 


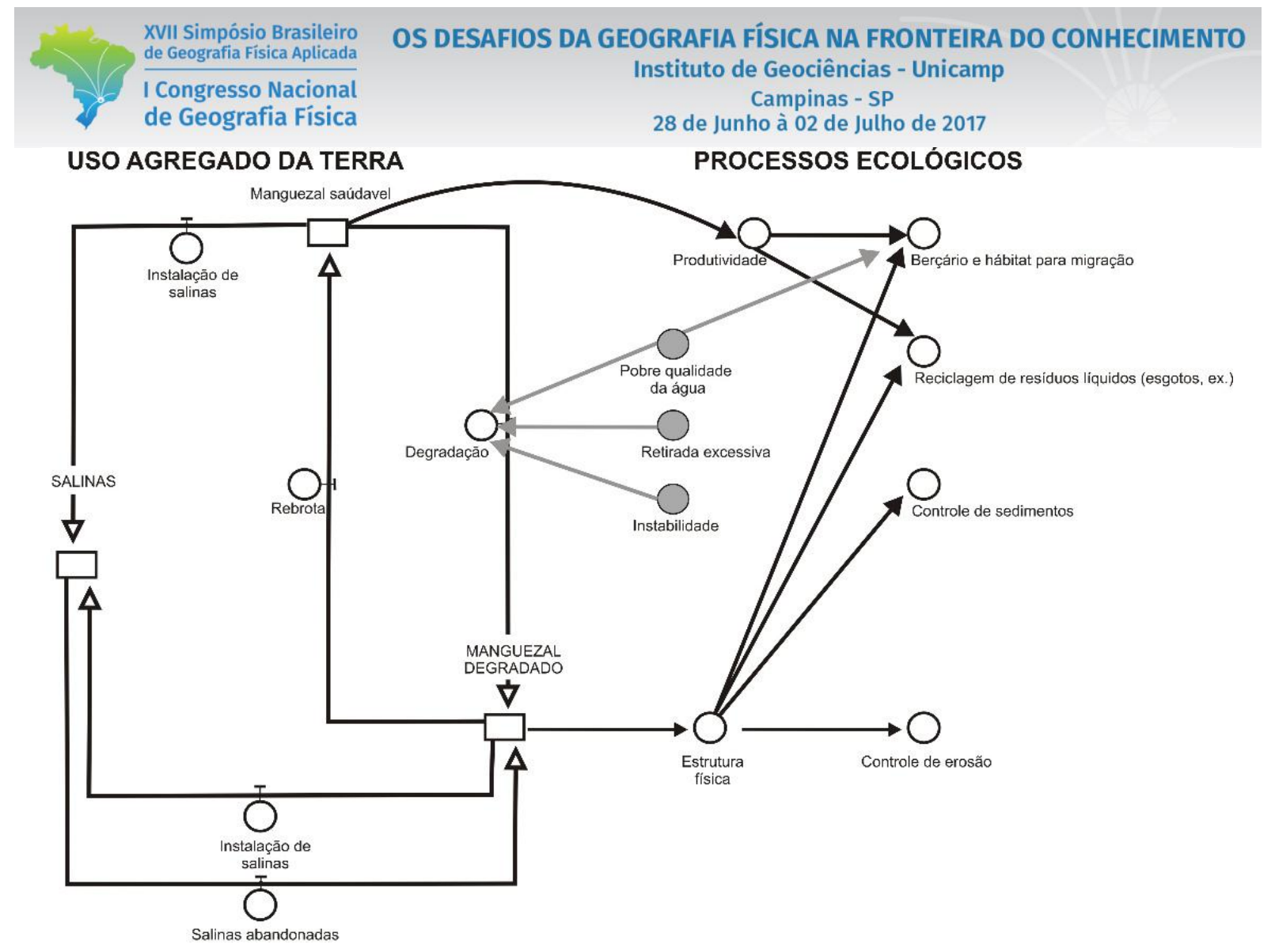

Figura 04 - Dinâmica agregada de uso humano e seus reflexos nos processos ecológicos do manguezal. Fonte: Adaptado de Gilbert \& Jansen (1998).

Em relação ao processo de aumento da estrutura física da vegetação (variável "estrutura física da vegetação"), ressalta-se que esta é largamente determinada pelo sistema de raízes aéreas e submersas (TOMLINSON, 1986), a qual é responsável pelo funcionamento de importantes serviços ecossistêmicos, os quais necessitam de uma análise integrada. Primeiramente, devido a grande quantidade de raízes, temse a retardação do fluxo de água, influenciando diretamente no controle de sedimentos (serviço de regulação) e mantendo a umidade no ambiente (SCHAEFFER-NOVELLI et al., 2000). Essa redução da velocidade da água cria um ambiente calmo e proporciona um habitat ideal, principalmente para espécies aquáticas juvenis (serviço de provisão) (WALTERS et al., 2008). Por fim, a passagem dos efluentes junto aos organismos fixados nas raízes do manguezal proporciona a reciclagem da água (serviço de regulação). Quanto aos Serviços de Provisão associados ao uso sustentável dos manguezais, este se dá principalmente para a construção de barcos eretirada de folhas para alimentação de animais (bovinos e caprinos). Todavia, é necessário que estes usos se dêem acompanhados de efetiva fiscalização e monitoramento, uma vez que a sobre-exploração destes serviçosatravés do "corte excessivo" também gera uma rede de 
retroalientação, estimulando a degradação do ecossistema, a qual traz custos para os usuários, por exemplo, em maior esforço de coleta de propágulos/sementes para os projetos de reflorestamento.

\section{Conclusões}

A utilização do sistema de diagramas mostrou-se uma ferramenta potencial na modelagem dos elos ecológicos-econômicos observados nos manguezais analisados, permitindo a estruturação de evidências que fornecem argumentos para manutenção dos serviços ecossistêmicos prestados por estes ecossistemas. Os processos ecológicos que são comuns para mais de um serviço ilustram claramente as interconexões entre os ecossistemas marinho, estuarino e o manguezal. Nesse sentido, a avaliação de algum programa de manejo e uso da terra nas margens dos estuários deve ser pautada em estratégias que assegurem a sustentabilidade do funcionamento desses ecossistemas, com vistas na manutenção dos serviços por eles prestados para o bem estar das comunidades que vivem no seu entorno. Também deve ser levado em consideração o fato de que, se um processo ecológico é comprometido, a repercussão poderá ser sentida por uma larga escala de usuários (humanos ou não).

Ao mesmo tempo, se não forem conhecidas estas interações, aumenta-se a chance de que uma decisão inapropriada possa levar a danos econômicos e ecológicos substanciais. Nesse sentido, espera-se que a partir das informações obtidas neste estudo de caso possam contribuir para o desenvolvimento das pesquisas sobre as interfaces existentes entre os sistemas ecológicos e os sistemas produtivos.

Deve-se destacar a existência de uma considerável incerteza em relação à interação dos diversos elementos constitutivos dos manguezais. Nessas circunstâncias, programas direcionados para a redução dos impactos - como a implantação de zonas tampão e o próprio replantio minimizarão as potenciais perdas econômicas. Portanto, a análise desenvolvida demonstra que o aperfeiçoamento das informações acerca da natureza destas inter-relações é essencial para que uma estratégia ecológica e economicamente viável possa ser estipulada.

\section{Agradecimentos}

Ao CERES - Centro de Ensino Superior do Seridó e ao LAMMA - Laboratório Multiusuário de Monitoramento Ambiental (UFRN - Campus de Caicó), pelo apoio logístico e instrumental, assim como a PROPESQ/UFRN (PVF10463-2014) e ao CNPq, pelo apoio financeiro no âmbito do projeto "Caracterização geoambiental e serviços ecossistêmicos prestados por áreas úmidas hipersalinas do Brasil (RN/CE)" (MCTI/CNPQ/Universal Proc.447227/2014-9). 


\section{Bibliografia}

ANDRADI-BROWN, D.A.; HOWE, C.; MACE, G.M.; KNIGHT, A.T. Do mangrove forest restoration or rehabilitation activities return biodiversity to pre-impact levels? Environmental Evidence, v. 2:20, 2013. doi:10.1186/2047-2382-2-20

COSTANZA, Robert et al. Changes in the global value of ecosystem services. Global Environmental Change, v. 26, p. 152-158, 2014.

FISHER, B.; TURNER, K.R.; MORLING, P. Defining and classifying ecosystem services for decision making.Ecol. Econ., v. 68, n. 3, p. 643-653, 2009.

GILBERT, A. J.; JANSSEN, R. Use of environmental functions to communicate the values of a mangrove ecosystem under different management regimes.Ecological Economics, v. 25, 1998, p. 323-346.

DE GROOT, R. S.; WILSON, M. A.; BOUMANS, R. M.J.A typology for the classification, description and valuation of ecosystem functions, goods and services.Ecological Economics, n. 41, 2002, p. 393-408.

DE GROOT, R.S.; ALKAMADE, R.; BRAAT, L.; HEIN, L.; WILLEMEN, L. Challenges in integrating the concept of ecosystem services and values in landscape planning, management and decision making. Ecological Complexity, v. 7, p, 260-272, 2010.

GRASSO, M. Ecological-economic model for optimal mangrove trade off between forestry and fishery production: comparing a dynamic optimization and a simulation model, Ecological Modeling, v. 112, 1998, p. 131-150.

GRASSO, M.; SCHAEFFER-NOVELLI, Y. Economic valuation of mangrove ecosystems. In: MAY, P.H. (org.) Natural resource valuation and policy in Brazil: methods and cases. Nova York: Columbia Univ. Press, 1999.

HAINES-YOUNG, Roy; POTSCHIN, Marion.Common International Classification of Ecosystem Services (CICES): Consultation on Version 4, August-December. EEA - Framework Contract, n. EEA/IEA/09/003. Nottingham: University of Nottingham/Centre for Environmental Management, 2013.

KJERFVE, B; LACERDA, L. D. Mangroves of Brazil.In: LACERDA, L. D. (ed.) Mangrove ecosystem studies in Latin America and Africa. International Society for Mangrove Ecosystem, Technical reports, v.2, 1993, p. 245275 .

LACERDA, L.D. Trace metals in mangrove plants: why such low concentrations? In: KJERFVE, B.; LACERDA, L.D.; DIOP, H.S. (Eds.). Mangrove Ecosystem Studies in Latin America and Africa. Paris: UNESCO, 1997, p.171-178.

LEE, S. Y. et al. Ecological role and services of tropical mangrove ecosystems: a reassessment. Global Ecology and Biogeography, v. 23, p. 726-743, 2014.

LUGO, A. E.; SNEDAKER, S. C.The ecology of mangroves, Annual Review of Ecology and Systematics, v. 5, 1974, p. 39-64.

MAES, J.; CROSSMAN, N.D.; BURKHARD, B. Mapping ecosystem services. In: POTSCHIN, P.; HAINESYOUNG, R.; FISH, R.; TURNER, R.K. (Eds). Routledge Handbook of Ecosystem Services. London: Routlegde, 2016, p. 188-204.

MAIA, L. P.; LACERDA, L. D. (orgs.). Estudo das áreas de manguezais do nordeste do Brasil - Avaliação das áreas de manguezais dos Estados do Piauí, Ceará, Rio Grande do Norte, Paraíba e Pernambuco. Fortaleza: Universidade Federal do Ceará. Instituto de Ciências do Mar, 2005, 60 pg.

MAITI, S.K.; CHOWDHURY, A. Effects of anthropogenic pollution on mangrove biodiversity: a review. Journal of Environmental Protection, v. 4, p. 1428-1434, 2013.

MARCHAND, C.; FERNANDEZ, J.M.; MORETON, B.; LANDI, L.; LALLIER-VERG ES, E. et al.The partitioning of transitional metals $(\mathrm{Fe}, \mathrm{Mn}, \mathrm{Ni}, \mathrm{Cr})$ in mangrove sediments downstream of a ferralitised ultramafic watershed (New Caledonia).Chemical Geology, v. 300-301, p.70-80, 2012.

MOTTA, R. S. Economia ambiental. Rio de Janeiro: FGV, 2006. 


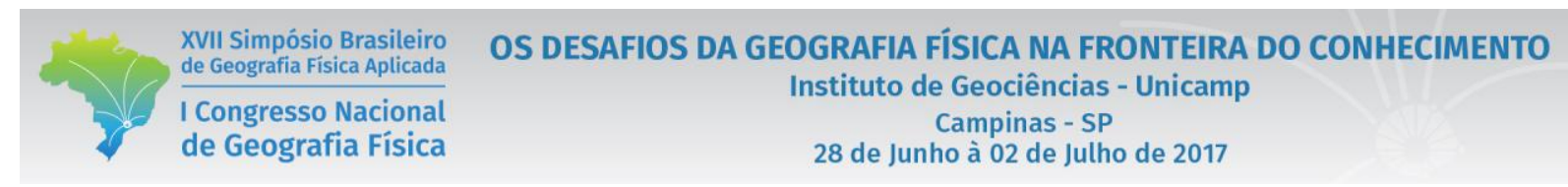

MUKHERJEE, N. et al. Using expert knowledge and modeling to define mangrove composition, functioning, and threats and estimate time frame for recovery.Ecology and Evolution, v. 4, n. 11, p. 2247-2262, 2014.

PRIMAVERA, J. H. Philippine mangroves: status, threats and sustainable development. In: VANNUCCI, M. (ed.) Mangrove management and conservation: Present and Future. New York: United Nations University Press, 2004.

RAMOS E SILVA, C. A.; SILVA, A.P.; OLIVEIRA, S.R. Concentration, stock and transport rate of heavy metals in a tropical red mangrove, Natal, Brazil, Marine Chemistry, v. 99, 2006, p. 2 -11.

RAMOS E SILVA, C. A.; OLIVEIRA, S.R.; RÊGO, R. D. P.; MOZETO, A. A. Dynamics of phosphorus and nitrogen through litter fall and decomposition in a tropical mangrove forest.Marine Environmental Research, v. 64, 2007, p. 524-534.

RAMOS E SILVA, C.A.; MIRANDA, L.B.; DÁVALOS, P.B.; PEREIRA DA SILVA, M. Hydrochemistry in tropical hyper-saline and positive estuaries. Pan-American Journal of Aquatic Sciences, v. 5, n. 3, p. 432-443, 2010.

SCHAEFFER-NOVELLI, Y.; CINTRÔN-MOLERO, G.; SOARES, M. L. G.; DE-ROSA, T. Brazilian mangroves.Aquatic Ecosystem Health and Management, v. 3, 2000, p. $561-570$.

TOMLINSON, P.B. The botany of mangroves. Cambridge: Cambridge University Press, 1986.

WALTERS, B. B.; RÖNNBÄCK, P.; KOVACS, J. M.; CRONA, B.; HUSSAIN, S. A.; BADOLA, R.; PRIMAVERA, J. H.; BARBIER, E.; DAHDOUH-GUEBAS, F. Ethnobiology, socio-economics and management of mangrove forests: a review. Aquatic Botany, n. 89, 2008, p. 220-236. 